\title{
Identity graphs of finite cyclic groups
}

\author{
Nazmiye Feyza YALÇIN ${ }^{1, *}$, Yakup KIRĞIL ${ }^{2}$ \\ ${ }^{1}$ Harran University, Faculty of Arts and Sciences, Department of Mathematics, Şanllurfa, Turkey \\ ${ }^{2}$ Harran University, Institute of Science, Şanlıurfa, Turkey \\ Gelis Tarihi (Received Date): 27.03.2019 \\ Kabul Tarihi (Accepted Date): 25.10.2019
}

\begin{abstract}
In this paper, identity graphs of finite cyclic groups are considered. The identity graphs of finite cyclic groups are examined regarding to the subset of self-inverse elements and the subset of mutual inverse elements in a group. By using the features of these subsets the number of triangles and the number of edges in the identity graphs of finite cyclic groups are determined. Furthermore, Schultz, Gutman, first Zagreb, second Zagreb and Wiener indices are computed for identity graphs.
\end{abstract}

Keywords: Graph, identity graph, cyclic group, topological index.

\section{Sonlu devirli grupların birim grafları}

$\ddot{\mathbf{O} z}$

Bu çalışmada sonlu devirli grupların birim grafları göz önüne alınmıştır. Sonlu devirli grupların birim graflarl grupta tersi kendisi olan elemanların alt kümesi ve tersi kendisinden farkl elemanların alt kümesi ile ilişkili olarak incelenmişsir. Bu alt kümelerin özellikleri kullanılarak sonlu devirli grupların birim graflarındaki üçgen sayısı ve kenar sayısı belirlenmiştir. Ayrıca birim grafların Schultz, Gutman, birinci Zagreb, ikinci Zagreb ve Wiener indeksleri hesaplanmuştır.

Anahtar kelimeler: Graf, birim graf, devirli grup, topolojik indeks.

\footnotetext{
* Nazmiye Feyza YALÇIN, fyalcin@ harran.edu.tr, https://orcid.org/0000-0001-5705-8658

Yakup KIRĞIL, yakupkirgil10@gmail.com, http://orcid.org/0000-0002-2300-0498
} 


\section{Introduction}

In recent years, graph theory and its applications associated to algebraic structures are studied extensively (see [1,2-7]). In [7], Kandasamy and Smarandache defined identity graphs of groups and semigroups and examined some special subgraphs. Let $G$ be a group and $x, y \in G, x$ is adjacent to $y$ if and only if $x y=e$, where $e$ is the identity element of $G$. Further, it is assumed that every element of $G$ is adjacent to $e$. If $x$ is adjacent to $y$, it is denoted by $x \sim y$ or $x-y$. By this adjacency, all elements of the group take place in the forming graph is called the identity graph of the group $G$ and denoted by $G_{I}(G)$. Thus all the elements of a group can be represented by a graph. From the adjacency definition, all of self-inverse and mutual inverse elements of a group can be seen directly from the identity graph of a group.

Let $G=(V(G), E(G))$ be a simple graph, where $V(G)$ is the set of all vertices called vertex set and $E(G)$ is the set of all edges which join the vertices called edge set. In a graph degree of a vertex $u$ is the number of edges incident to $u$ and denoted by deg $u$. If there is a path between any two distinct vertices of a graph, then the graph is called connected. The shortest path connecting the vertices $u$ and $v$ is called distance between $u$ and $v$, denoted by $d(u, v)$. The diameter of a graph $G$ is the greatest distance between any pair of vertices, i.e. $\operatorname{diam}(G)=\max \{d(u, v): u, v \in V(G)\}$. The girth of a simple graph $G$ is the length of a smallest cycle and denoted by $\operatorname{gir}(G)$.

Topological indices are graph invariants that are mostly based on the distances between the vertices of a graph and independent from labeling and visual representation of graphs. Let $G=(V(G), E(G))$ be a simple connected finite graph. In [4], degree distance of a graph $G$ is defined as

$D^{\prime}(G)=\sum_{v \in V(G)}$ degv. $D_{G}(v)$,

where $D_{G}(v)=\sum_{u \in V(G)} d(u, v)$ is called distance of a vertex $v$ in $G$. Degree distance of a graph renamed as "Schultz index" which is proposed by Gutman in [6]. Schultz index can be also expressed as

$D^{\prime}(G)=\sum_{\{u, v\} \subseteq V(G)}[\operatorname{deg} u+\operatorname{deg} v] d(u, v)$.

Schultz index of the second kind is introduced in [6] and called as Gutman index. It is defined as

$\operatorname{Gut}(G)=\sum_{\{u, v\} \subseteq V(G)}[\operatorname{degu} . \operatorname{deg} v] d(u, v)$.

First Zagreb and second Zagreb indices of a graph $G$ are introduced by Gutman and Trinajstic in [5] and defined respectively as

$M_{1}(G)=\sum_{u \in V(G)}(\text { degu })^{2}$,

$M_{2}(G)=\sum_{u v \in E(G)}$ degu.degv.

One of the oldest distance-based topological index is Wiener index which is equal to the sum of distances between all unordered pairs of vertices of a graph $G$ and and defined in [8] as 
$W(G)=\sum_{\{u, v\} \subseteq V(G)} d(u, v)$

Schultz index also can be considered as degree-weighted version of the Wiener index.

\section{Preliminaries}

In this section some required definitions and theorems are presented. Moreover some explicit results are given for the identity graphs of finite cyclic groups.

\subsection{Definition}

Let $(G, *)$ be a finite group with the identity element $e$ and $a \in G$. If $a=a^{-1}$, then $a \in$ $G$ is called self-inverse element of $G$. All of the self-inverse elements of $G$ is denoted by $S(G)$

\subsection{Definition ([3])}

Let $(G, *)$ be a finite group with the identity element $e$ and $a \in G$. If there exists $b \in G$ such that $a * b=b * a=e$, then $a \in G$ is called mutual inverse element of $G$. All of these elements of $G$ is denoted by $M(G)$.

According to the above definitions, $S(G)$ and $M(G)$ can be expressed as follows.

$S(G)=\left\{a \in G: a=a^{-1}\right\}$, $M(G)=\left\{a \in G: a \neq a^{-1}\right\}$.

For a finite group $G$, obviously we have $G=S(G) \cup M(G)$ and $S(G) \cap M(G)=\emptyset$.

\subsection{Theorem ([3])}

If $G$ be a finite cyclic group, then

$|S(G)|=\left\{\begin{array}{ll}1, & \text { if }|G| \text { is odd } \\ 2, & \text { if }|G| \text { is even }\end{array}\right.$,

where | | denotes the cardinality of $S(G)$.

\subsection{Corollary ([3])}

If $G$ be a finite group of even order, then $|S(G)|$ and $|M(G)|$ are both even order.

\subsection{Remark ([3])}

If $G$ is not a cyclic group, then $|S(G)| \geq 2(p .129, E x .1-2)$.

\subsection{Corollary ([3])}

If $G$ be a finite group of odd order, then $|S(G)|=1$ and $|M(G)|=|G|-1$.

\subsection{Remark}

Let $G=\langle a\rangle$ be a cyclic group of order $n$ and $k \in Z$. If $a^{k} \in M(G)$, then obviously we have $n \nmid 2 k$ and if $a^{k} \in S(G)$, then $n \mid 2 k$. 
Kandasamy and Smarandache in [7], determine the number of triangles in identity graphs of finite cyclic groups in the following theorems.

\subsection{Theorem ([7])}

If $G=<a>$ be a cyclic group of order $p$, where $p$ is a prime, then $G_{I}(G)$. is formed by only $\frac{p-1}{2}$ triangles.

\subsection{Corollary}

Let $n$ is an odd number. If $G=\left\langle a>\right.$ be a cyclic group of order $n$, then $G_{I}(G)$ is formed by only $\frac{n-1}{2}$ triangles.

According to the Corollary 2.3, since degree of an any vertex is even in the identity graph of finite group of odd order, we can give the following result.

\subsection{Corollary}

If $G$ is a cyclic group of odd order, then $G_{I}(G)$ is Eulerian.

\subsection{Theorem ([7])}

Let $n$ is an even number. If $G=<a>$ be a cyclic group of order $n$, then $G_{I}(G)$ is formed by $\frac{n-1}{2}$ triangles and a line.

\subsection{Corollary}

If $G$ is a cyclic group of even order, then $G_{I}(G)$ is not Eulerian.

\subsection{Corollary}

If $G$ be a finite cyclic group, then $\operatorname{gir}\left(G_{I}(G)\right)=3$.

\section{Proof.}

The girth of a simple graph is the length of a smallest cycle. Since $G_{I}(G)$ has always formed by triangles (3-cycle), we have $\operatorname{gir}\left(G_{I}(G)\right)=3$.

The main purpose of this paper is to examine some properties and computing some topological indices of the identity graphs of finite cyclic groups by using the features of $S(G)$, the subset of self-inverse elements and $M(G)$, the subset of mutual inverse elements.

\section{Main results}

In this section, initially we express some fundamental properties of the identity graphs of finite cyclic groups by using the cardinality of the disjoint sets $S(G)$ and $M(G)$. Moreover, we compute Schultz, Gutman, first Zagreb, second Zagreb and Wiener index of the identity graphs of a finite cyclic groups.

Let $G$ be a cyclic group of order $n$ with identity $e$ and consider $G_{I}(G)$, the identity graph of $G$. Obviously we have $V\left(G_{I}(G)\right)=G$ and from the adjacency of the identity graph, we have $\operatorname{deg}(e)=n-1$. 


\subsection{Theorem}

Let $G$ be a finite group. Then $G_{I}(G)$ is a connected graph.

\section{Proof.}

The proof is obvious from the adjacency of the identity graph $G_{I}(G)$.

\subsection{Theorem}

Let $G=\langle a>$ be a cyclic group of even order. If $u \in S(G) \backslash\{e\}$, then we have degu= 1.

\section{Proof.}

If $|G|$ is even, then from Theorem $2.1,|S(G)|=2$ and we have $S(G)=\left\{e, a: a^{2}=e\right\}$. So, if $u \in S(G) \backslash\{e\}$, then $u$ is only adjacent to $e$ and $u$ is a pendant vertex. Thus proof is completed.

Recall that the number of triangles are given in [7]. Now we give a brief proof by using the cardinality of $M(G)$.

\subsection{Theorem}

Let $G$ be a finite cyclic group. If $u \in M(G)$, then $\operatorname{deg}(u)=2$ and $t=\frac{|M(G)|}{2}$, where $t$ is the number of triangles in the identity graph $G_{I}(G)$.

\section{Proof.}

If $u \in M(G)$, then $u \neq u^{-1}$ and from the adjacency, we have $u \sim u^{-1}, u \sim e$ and $u^{-1} \sim e$. Thus, $\operatorname{deg}(u)=2$. For any $u \in M(G)$, each $\left\{u, u^{-1}\right\}$ pair of vertices forms a triangle in $G_{I}(G)$. So, there are $t=\frac{|M(G)|}{2}$ triangles in $G_{I}(G)$ and proof is completed.

\subsection{Corollary}

Let $G$ be a cyclic group of order $n$ and $t$ be the number of triangles in the identity graph $G_{I}(G)$. Then we have

$t=\left\{\begin{array}{ll}\frac{n-1}{2}, & \text { if } n \text { is odd } \\ \frac{n-2}{2}, & \text { if } n \text { is even }\end{array}\right.$.

\section{Proof.}

The proof can be seen directly by Theorem 3.3, Corollary 2.1 and Corollary 2.2.

\subsection{Example}

Let $G=\langle a>$ be a cyclic group of order 7 and $H=<b>$ be a cyclic group of order 8 . Then $M(G)=\left\{a, a^{2}, a^{3}, a^{4}, a^{5}, a^{6}\right\}, S(G)=\{e\}$ and $M(H)=\left\{b, b^{2}, b^{3}, b^{5}, b^{6}, b^{7}\right\}$, $S(H)=\left\{e, b^{4}\right\}$. The identity graphs of the groups $G$ and $H$ are given respectively in Fig. 1. From Fig. 1, each $\left\{u, u^{-1}\right\}$ pair of vertices of mutual inverse elements of the group forms a triangle and if the order of the group is an even integer, then self-inverse elements of the group form a line in the identity graph. 


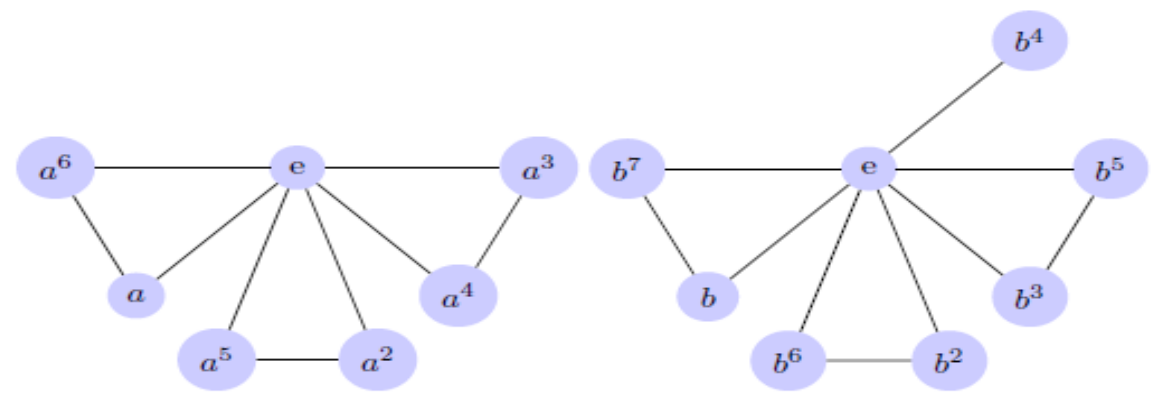

Figure 1. $G_{I}(G)$ and $G_{I}(H)$ identity graphs of the cyclic groups $G$ and $H$.

\subsection{Theorem}

If $G$ be a cyclic group of order $n$, then we have $\left|E\left(G_{I}(G)\right)\right|=\left\{\begin{array}{cl}\frac{3(n-1)}{2}, & \text { if } n \text { is odd } \\ \frac{3(n-2)}{2}+1, & \text { if } n \text { is even }\end{array}\right.$

\section{Proof.}

Let $|G|=n$ is even, then from Theorem 2.1, we have $|S(G)|=2$, so $|M(G)|=n-2$. For $e \in S(G)$ and $u \in S(G) \backslash\{e\}$, obviously we get $\operatorname{deg}(e)=n-1$ and $\operatorname{deg}(u)=1$. Thus we have $\operatorname{deg}(e)+\operatorname{deg}(u)=n$. Since the other $n-2$ vertices different from $e$ and $u$ in the identity graph $G_{I}(G)$ belong to $M(G)$ and for any $v \in M(G), \operatorname{deg}(v)=2$, we have

$\sum_{a \in G} \operatorname{deg} a=n+2(n-2)=3 n-4=2\left|E\left(G_{I}(G)\right)\right|$.

Thus, proof is complete. If we consider that $|M(G)|=n-2$, we can also write $\left|E\left(G_{I}(G)\right)\right|=3 t+1$. For $n$ is an odd integer, the proof can be seen similarly.

\subsection{Theorem}

If $G=<a>$ be a cyclic group of order $n$, then $\operatorname{diam}\left(G_{I}(G)\right)<3$.

\section{Proof.}

If $n=2$, then we have $S(G)=\left\{e, a: a^{2}=e\right\}$ and $M(G)=\emptyset$. From adjacency of the identity graph, $G_{I}(G)$ is exactly formed by a line $e-a$. So, $\operatorname{diam}\left(G_{I}(G)\right)=1$. If $n=$ 3 , then from Theorem $2.2 G_{I}(G)$ is exactly a triangle. Thus $\operatorname{diam}\left(G_{I}(G)\right)=1$. Let $n>3$. If $n$ is even, then we have $S(G)=\left\{e, a: a^{2}=e\right\},|M(G)|=n-2$ and from Theorem 2.3, $G_{I}(G)$ is formed by $\frac{n-1}{2}$ triangles and a line $a-e$. If $b \in M(G)$, since $a \in S(G) \backslash\{e\}$, there is always a shortest $a-e-b$ path of length 2 , we have $\operatorname{diam}\left(G_{I}(G)\right)=2$. If $b, c \in M(G)$ and $b \sim c$, then $d(b, c)=1$. If $b \nsim c$, namely $b$ is not adjacent to $c$, then there is always a shortest $b-e-c$ path of length 2 . Thus $\operatorname{diam}\left(G_{I}(G)=2\right.$. If $n$ is odd, we have $S(G)=\{e\}$ and $|M(G)|=n-1$ by Corollary 2.2. From Corollary 2.3, $G_{I}(G)$ is formed by only triangles. Let $b, c \in M(G)$ are in different triangles, then there is always a shortest $b-e-c$ path of length 2 . So, we have $\operatorname{diam}\left(G_{I}(G)\right)=2$ and proof is completed.

\subsection{Theorem}

Let $G$ be a cyclic group of order $n$. Schultz index of $G_{I}(G)$ is 
$D^{\prime}\left(G_{I}(G)\right)=\left\{\begin{array}{l}5 n^{2}-14 n+9, \quad \text { if } n \text { is odd } \\ 5 n^{2}-16 n+14, \text { if } n \text { is even }\end{array}\right.$.

\section{Proof.}

We consider two cases for proof as follows.

Case 1. Let $n$ is odd. Then from Corollary 2.2, we have $S(G)=\{e\}$ and $|M(G)|=$ $n-1$. Thus Schultz index of $G_{I}(G)$ is

$$
\begin{aligned}
& D^{\prime}\left(G_{I}(G)\right)=\sum_{\{u, v\} \subseteq V(G)}[\operatorname{deg} u+\operatorname{deg} v] d(u, v) \\
& =\sum_{u \sim e ; u \in M(G)}[\operatorname{deg} u+\operatorname{deg}] .1+\sum_{u \sim u^{-1} ; u \in M(G)}\left[\operatorname{deg} u+\operatorname{deg}\left(u^{-1}\right)\right] .1 \\
& +\sum_{u \nsim v ; u, v \in M(G)}[\operatorname{deg} u+\operatorname{deg} v] .2 .
\end{aligned}
$$

Since $|M(G)|=n-1$ and any vertex in $M(G)$ is adjacent to $e$, contribution of the first sum is $(n-1)(2+(n-1))$. Consider the second sum. If $u, u^{-1} \in M(G)$, then we get $\operatorname{deg} u=\operatorname{deg}\left(u^{-1}\right)=2$ and $u \sim u^{-1}, u \sim e$ and $u^{-1} \sim e$. Therefore contribution of the second sum is $4 t$, where $t$ is the number of triangles, that is $2(n-1)$ from Corollary 3.1. In the last sum, we require the number of paths of length 2. Obviously, the number of paths of length 1 is the number of edges, that is $\frac{3(n-1)}{2}$. If we consider the distance matrix of a graph with $n$ vertices, the number of entries above the main diagonal is $\frac{n^{2}-n}{2}$. Therefore the number of paths of length 2 is

$$
\begin{aligned}
& \frac{n^{2}-n}{2}-\left|E\left(G_{I}(G)\right)\right|=\frac{n^{2}-n}{2}-\frac{3(n-1)}{2} \\
& =\frac{(n-1)(n-3)}{2} .
\end{aligned}
$$

If we write these contributions in (1), we have

$D^{\prime}\left(G_{I}(G)\right)=(n-1)(n+1)+2(n-1)+8 \cdot \frac{(n-1)(n-3)}{2}$

$=(n-1)(5 n-9)$

$=5 n^{2}-14 n+9$.

Case 2. Let $n$ is even. Then by Theorem 2.1, we have $|S(G)|=2$ and $|M(G)|=n-2$. So, we get

$$
\begin{aligned}
& D^{\prime}\left(G_{I}(G)\right)=\sum_{\{u, v\} \subseteq V(G)}[\text { degu }+ \text { degv }] d(u, v) \\
& =\sum_{u \sim e ; u \in M(G)}[\text { degu }+ \text { dege }] .1+\sum_{u \sim e ; u \in S(G) \backslash\{e\}}[\text { degu+dege }] .1 \\
& +\sum_{u \sim u^{-1} ; u \in M(G)}\left[\operatorname{deg} u+\operatorname{deg}\left(u^{-1}\right)\right] .1 \\
& +\sum_{u \in M(G) ; v \in S(G) \backslash\{e\}}[\operatorname{deg} u+\operatorname{deg} v] .2+\sum_{u \times v ; u, v \in M(G)}[\text { degu }+ \text { deg } v] .2 .
\end{aligned}
$$


Similar to the Case 1, contribution of the first three sum is

$$
(n-2)(n+1)+n+4\left(\frac{n-2}{2}\right) \text {. }
$$

Consider the sum $\sum_{u \in M(G) ; v \in S(G) \backslash\{e\}}[$ degu + degv $]$. 2. Obviously we have degu $=2$ and $\operatorname{deg} v=1$. Since $v \in S(G) \backslash\{e\}$ is only adjacent to $e, v$ is not adjacent to any vertex of $M(G)$. Thus the distance from the vertex $v$ to $n-2$ vertices of $M(G)$ is 2 and so, the number of paths of length 2 is $n-2$. Similar to the Case 1 , the total number of paths of length 2 is

$$
\begin{aligned}
& \frac{n^{2}-n}{2}-\left|E\left(G_{I}(G)\right)\right|=\frac{n^{2}-n}{2}-\left[\frac{3(n-2)}{2}+1\right] \\
& =\frac{(n-2)^{2}}{2} .
\end{aligned}
$$

Now, consider the sum $\sum_{u \times v ; u, v \in M(G)}[\operatorname{deg} u+\operatorname{deg} v]$. 2. For $u, v \in M(G)$, the number of paths of length 2 is

$$
\frac{(n-2)^{2}}{2}-(n-2)=\frac{(n-2)(n-4)}{2} \text {. }
$$

If we substitute the values in (2) and by (3), we have

$D^{\prime}\left(G_{I}(G)\right)=(n-2)(n+1)+n+4 \frac{(n-2)}{2}+6(n-2)+8 \frac{(n-2)(n-4)}{2}$

$=(n-2)(5 n-7)+n$

$=5 n^{2}-16 n+14$.

So, proof is complete.

\subsection{Theorem}

If $G$ be a cyclic group of order $n$, then the first Zagreb index of $G_{I}(G)$ is

$M_{1}\left(G_{I}(G)\right)=\left\{\begin{array}{cl}n^{2}+2 n-3, & \text { if } n \text { is odd } \\ n^{2}+2 n-6, & \text { if } n \text { is even }\end{array}\right.$.

\section{Proof.}

Let $n$ is even. Then $|S(G)|=2$ and $|M(G)|=n-2$. The first Zagreb index of $G_{I}(G)$ is

$$
\begin{aligned}
& M_{1}\left(G_{I}(G)\right)=\sum_{u \in G}(\text { degu })^{2} \\
& =\sum_{u \in S(G)}(\text { degu })^{2}+\sum_{u \in M(G)}(\text { degu })^{2} \\
& =1+(\text { dege })^{2}+4|M(G)| \\
& =1+(n-1)^{2}+4(n-2) \\
& =n^{2}+2 n-6,
\end{aligned}
$$


which completes proof. Proof can be seen similarly when $n$ is an odd integer.

\subsection{Theorem}

If $G$ be a cyclic group of order $n$, then the second Zagreb index of $G_{I}(G)$ is

$M_{2}\left(G_{I}(G)\right)=\left\{\begin{array}{cl}2 n^{2}-2 n, & \text { if } n \text { is odd } \\ 2 n^{2}-3 n-1, & \text { if } n \text { is even }\end{array}\right.$.

\section{Proof.}

If $n$ is an even integer, then the second Zagreb index of $G_{I}(G)$ is

$$
\begin{aligned}
& M_{2}\left(G_{I}(G)\right)=\sum_{u v \in E(G)} \text { degu.degv } \\
& =\sum_{u \sim e ; u \in M(G)} \text { degu.dege }+\sum_{\substack{u \sim e ; u \in S(G) \backslash\{e\}\\
}} \text { degu.dege } \\
& =\sum_{u \sim u^{-1} ; u \in M(G)}\left[\text { degu.deg }\left(u^{-1}\right)\right] .1 \\
& =2(n-2)(n-1)+(n-1)+4 t \\
& \left.=2 n^{2}-3 n-1\right)(n-1)+(n-1)+2(n-2)
\end{aligned}
$$

Thus proof is completed.

\subsection{Theorem}

If $G$ be a cyclic group of order $n$, then we have

$$
W\left(G_{I}(G)\right)=\left\{\begin{array}{ll}
\frac{2 n^{2}-5 n+3}{2}, & \text { if } n \text { is odd } \\
\frac{2 n^{2}-5 n+4}{2}, & \text { if } n \text { is even }
\end{array} .\right.
$$

\section{Proof.}

Let $n$ is odd. If we use the number of paths in the proof of Theorem 3.6, then we have

$$
\begin{aligned}
& W\left(G_{I}(G)\right)=\sum_{\{u, v\} \subseteq G} d(u, v) \\
& =\sum_{u \sim e ; u \in M(G)} d(u, e)+\sum_{\substack{u \sim u^{-1} ; u \in M(G) \\
(n-1)(n-3)}} d\left(u, u^{-1}\right)+\sum_{u \times v ; u, v \in M(G)} d(u, v) \\
& =(n-1) .1+t .1+\frac{3(n-1)}{2}+(n-1)(n-3) \\
& =\frac{2}{2} .5 n+3 \\
& =\frac{2 n^{2}-5}{2} .
\end{aligned}
$$

Now let $n$ is even. Then we also get 


$$
\begin{aligned}
& W\left(G_{I}(G)\right)=\sum_{u \sim e ; u \in M(G)} d(u, e)+\sum_{u \sim u^{-1} ; u \in M(G)} d\left(u, u^{-1}\right)+\sum_{u \in M(G) ; v \in S(G) \backslash\{e\}} d(u, v)+\sum_{u \sim v ; u, v \in M(G)} d(u, v) \\
& =(n-2) \cdot 1+1+t .1+|M(G)| .2+\frac{(n-2)(n-4)}{2} \cdot 2 \\
& =n-1+\frac{n-2}{2}+2(n-2)+(n-2)(n-4) \\
& =\frac{2 n^{2}-5 n+4}{2},
\end{aligned}
$$

and proof is complete.

\section{References}

[1] Anderson, D.F. and Livingston, P.S., The zero-divisor graph of a commutative ring, Journal of Algebra, 217: 434-447, (1999).

[2] Anderson, D.F. and Badawi, A., The total graph of a commutative ring, Journal of Algebra, 320, 2706-2719, (2008).

[3] Chalapathi, T. and Kumar, R.V.M.S.S., Invertible graphs of finite groups, Computer Science Journal of Moldova, 26(2), 126-145, (2018).

[4] Dobrynin, A.A. and Kochetova, A.A., Degree Distance of a Graph: A degree analogue of the Wiener index, Journal of Chemical Information and Computer Sciences, 34, 1082-1086, (1994).

[5] Gutman, I., and Trinajstic, N., Graph theory and molecular orbitals, Total $\varphi$-electron energy of alternant hydrocarbons, Chemical Physics Letters, 17, 535-538, (1972).

[6] Gutman, I., Selected properties of the Schultz molecular topological index, Journal of Chemical Information and Computer Sciences, 34, 1087-1089, (1994).

[7] Kandasamy, W.B.V. and Smarandache, F., Groups as Graphs, Editura CuArt 2009, http://www.gallup.unm.edu/ smarandache/eBooks-otherformats.htm

[8] Wiener, H., Structural determination of paraffin boiling points, Journal of the American Chemical Society, 69, 17-20, (1947). 